\title{
Weak Decays of Charmed Baryons
}

\author{
Fanrong $\mathbf{X u}^{*}$ \\ Department of Physics, Jinan University, Guangzhou 510632, People’s Republic of China \\ E-mail: fanrongxu@jnu.edu.cn
}

\begin{abstract}
The Cabibbo-favored (CF) and singly Cabibbo-suppressed (SCS) two-body hadronic weak decays of antitriplet charmed baryons $\Lambda_{c}^{+}, \Xi_{c}^{0}$ and $\Xi_{c}^{+}$are studied in this work. In the topologic diagram approach, both factorizable and nonfactorizable contributions are considered. The estimation of nonfactorizable contribution from $W$-exchange and inner $W$-emission diagrams relies on the pole model and current algebra. The non-perturbative parameters in both factorizable and nonfactorizable parts are all calculated in the MIT bag model. We calculate the branching fractions and up-down decay asymmetries for all the CF and SCS decays of these antitriplet charmed baryons. The prediction of $\mathscr{B}\left(\Xi_{c}^{+} \rightarrow \Xi^{0} \pi^{+}\right)$agrees well with the recent Belle result, while the calculated $\mathscr{B}\left(\Xi_{c}^{0} \rightarrow \Xi^{-} \pi^{+}\right)$is too large compared to the recent Belle measurement. We conclude that these two $\Xi_{c} \rightarrow \Xi \pi^{+}$modes cannot be simultaneously explained within the current-algebra framework for $S$-wave amplitudes. This issue needs to be resolved in future study. The long-standing puzzle with the branching fraction and decay asymmetry of $\Lambda_{c}^{+} \rightarrow \Xi^{0} K^{+}$is resolved. We find that not only the calculated rate agrees with experiment but also the predicted decay asymmetry is consistent with the SU(3)-flavor symmetry approach in sign and magnitude.
\end{abstract}

European Physical Society Conference on High Energy Physics - EPS-HEP2019 -

10-17 July, 2019

Ghent, Belgium

${ }^{*}$ Speaker. 


\section{Introduction}

Recently, there has been a significant progress in the experimental study of charmed baryon physics. The long-quested doubly charmed baryon was observed. Some breakthrough has also been made in singly charmed baryons as well, especially the lightest charmed baryon $\Lambda_{c}^{+}$. Both Belle and BESIII have measured the absolute branching fraction of the decay $\Lambda_{c}^{+} \rightarrow p K^{-} \pi^{+}$. A new average of $(6.28 \pm 0.32) \%$ for this benchmark mode is quoted by the Particle Data Group (PDG) The measurement of $\Lambda_{c}^{+} \rightarrow p \pi^{0}, p \eta$ indicated that singly Cabibbo-suppressed (SCS) decays could also be accessed. In addition to $\Lambda_{c}^{+}$, there have been some new progresses in the study of $\Xi_{c}^{0}$ and $\Xi_{c}^{+}$, the two other singly charmed baryons in the antitriplet. By using a data set comprising $(772 \pm 11) \times 10^{6} B \bar{B}$ pairs collected at $\Upsilon(4 S)$ resonance, Belle was able to measure the absolute branching fraction for $B^{-} \rightarrow \bar{\Lambda}_{c}^{-} \Xi_{c}^{0}$. Combining the subsequently measured product branching fractions such as $\mathscr{B}\left(B^{-} \rightarrow \bar{\Lambda}_{c}^{-} \Xi_{c}^{0}\right) \mathscr{B}\left(\Xi_{c}^{0} \rightarrow \Xi^{-} \pi^{+}\right)$, Belle reported the first weak decay of $\Xi_{c}^{0}$

$$
\mathscr{B}\left(\Xi_{c}^{0} \rightarrow \Xi^{-} \pi^{+}\right)=(1.80 \pm 0.50 \pm 0.14) \times 10^{-2} .
$$

Using the same technique, a channel of two-body weak decay with a vector meson in final state was also measured, $\mathscr{B}\left(\Xi_{c}^{+} \rightarrow p \bar{K}^{0 *}(892)\right)=(0.25 \pm 0.16 \pm 0.04) \times 10^{-2}$ It is worth pointing out that the absolute branching fraction for three-body decay was obtained by Belle to be $\mathscr{B}\left(\Xi_{c}^{+} \rightarrow\right.$ $\left.\Xi^{-} \pi^{+} \pi^{+}\right)=(2.86 \pm 1.21 \pm 0.38) \times 10^{-2}$, from which we can read

$$
\mathscr{B}\left(\Xi_{c}^{+} \rightarrow \Xi^{0} \pi^{+}\right)=(1.57 \pm 0.83) \% .
$$

after making use of $\Gamma\left(\Xi_{c}^{+} \rightarrow \Xi^{0} \pi^{+}\right) / \Gamma\left(\Xi_{c}^{+} \rightarrow \Xi^{-} \pi^{+} \pi^{+}\right)=(0.55 \pm 0.13 \pm 0.09)$ given by the CLEO.

Among the methodologies to calculate charmed baryon hadronic weak decays in theory, topological diagram approach is the one which can depict dynamical mechanism. From topological diagram, the nonfactorizable contribution from $W$-exchange or nonfactorizable internal $W$-emission diagrams plays an essential role and hence cannot be omitted, in contrast with the negligible effect in heavy meson decays. The fact that all the decays of $\Xi_{c}^{+, 0}$ receive contributions from the nonfactorizable terms, especially some decays such as $\Xi_{c}^{0} \rightarrow \Sigma^{+} K^{-}, \Xi^{0} \pi^{0}$ proceed only through purely nonfactorizable diagrams, will allow us to check the importance and necessity of nonfactorizable contributions. However, we still do not have a reliable phenomenological model to calculate charmed baryon hadronic decays so far. Various techniques were developed in the 1990s. Now with more experimental data accumulated, there are some updated studies in theory. A reconsideration of charmed baryon weak decays, revealing the dynamics at the quark level, is timely and necessary. Pole model is one of the choices.

In the pole model people usually consider two most important low-lying $1 / 2^{+}$and $1 / 2^{-}$states under the pole approximation. In the decay with a pseudoscalar in the final state, $\mathscr{B}_{c} \rightarrow \mathscr{B}^{\prime}+P$, the nonfactorizable $S$-wave amplitude and $P$-wave amplitude are dominated by $1 / 2^{-}$low-lying baryon resonance and $1 / 2^{+}$ground state baryon, respectively. In addition to a concrete calculation of $1 / 2^{-}$contribution in a particular quark model with the frame of pole model, the $S$-wave amplitude can be reduced to current algebra in the soft-pseudoscalar limit. The methodology was developed and applied in the earlier work [1]. Recently we follow this method to calculate singly 
Cabibbo-suppressed (SCS) decays of $\Lambda_{c}^{+}$[2], in which the predictions of $\Lambda_{c}^{+} \rightarrow p \pi^{0}, p \eta$ are in good agreement with the BESIII measurement. And in a continuing work [3], we further calculate CF and SCS processes of $\Xi_{c}$ baryons together with a latest update of CF processes of $\Lambda_{c}^{+}$. We summarize all the $\mathrm{CF}$ and CSC decay processes of the antitriplet charmed baryon in this proceeding.

This paper is organized as follows. In Sec. 2 we will describe how to calculate branching fraction and decay asymmetry, including contributions from both factorizable and nonfactorizable terms. Numerical results are presented in Sec.3. A conclusion will be given in Sec. 4.

\section{Framework}

The amplitude for the two-body weak decay $\mathscr{B}_{i} \rightarrow \mathscr{B}_{f} P$ generally can be parameterized as

$$
M\left(\mathscr{B}_{i} \rightarrow \mathscr{B}_{f} P\right)=i \bar{u}_{f}\left(A-B \gamma_{5}\right) u_{i}
$$

in which $P$ in the final state is a pseudoscalar meson. Based on the $S$ - and $P$ - wave amplitudes, $A$ and $B$, the decay width and up-down spin asymmetry are given as

$$
\begin{aligned}
& \Gamma=\frac{p_{c}}{8 \pi}\left[\frac{\left(m_{i}+m_{f}\right)^{2}-m_{P}^{2}}{m_{i}^{2}}|A|^{2}+\frac{\left(m_{i}-m_{f}\right)^{2}-m_{P}^{2}}{m_{i}^{2}}|B|^{2}\right], \\
& \alpha=\frac{2 \kappa \operatorname{Re}\left(A^{*} B\right)}{|A|^{2}+\kappa^{2}|B|^{2}},
\end{aligned}
$$

with $\kappa=p_{c} /\left(E_{f}+m_{f}\right)=\sqrt{\left(E_{f}-m_{f}\right) /\left(E_{f}+m_{f}\right)}$ and $p_{c}$ is the three-momentum in the rest frame of mother particle. The $S$ - and $P$ - wave amplitudes of the two-body decay, which reveal the dynamics, generically receive both factorizable and nonfactorizable contributions, giving

$$
A=A^{\mathrm{fac}}+A^{\mathrm{nf}}, \quad B=B^{\mathrm{fac}}+B^{\mathrm{nf}} .
$$

With the help of topological diagram, one can easily find whether a process receives part or both contributions. The external- $W$ emission diagram and inner- $W$ emission from the external quark are usually classified as factorization contribution, while the nonfactorizable contribution are remarked from inner $W$ emission from an inner quark as well as $W$ exchange diagram. It is interesting to realize that processes contain only pure factorizable contribution cannot be found in $\Xi_{c}^{+, 0}$ decays, contrary to weak decays of $\Lambda_{c}^{+}$,

The factorizable amplitude, obtained from the standard effective Hamiltonian approach, contains both perturbative and non-perturbative parameters. Among the non-perturbative parameters, the $\mathscr{B}_{c} \rightarrow \mathscr{B}$ baryon-baryon transition form factor (FF) plays a crucial role, which determines whether the interference with nonfactorizable amplitude is destructive or constructive. For the purpose of global consistence, at least for the consistence of sign, in this work these FFs are calculated in the frame of MIT bag model.

In pole model, we establish the connection between quark and hadron diagram, and hence nonfactorizable amplitude can be parameterized at hadron level. The excited baryon state $\frac{1}{2}-$ are involved in $S$-wave amplitude, bringing in complication for relevant calculation. With the technique of current algebra, $\frac{1}{2}^{-}$can be avoided and a commutator is induced. Finally the $S$-wave amplitude resorts to matrix elements of a set of commutators between conserved charge and Hamiltonian. As 
Table 1: The predicted $S$ - and $P$-wave amplitudes of Cabibbo-favored (upper entry) and singly Cabibbosuppressed (lower entry) $\Lambda_{c}^{+} \rightarrow \mathscr{B}+P$ decays in units of $10^{-2} G_{F} \mathrm{GeV}^{2}$. Branching fractions and the asymmetry parameter $\alpha$ are shown in the last four columns.

\begin{tabular}{lrrrrrr|rrrr}
\hline \hline Channel & $A^{\text {fac }}$ & $A^{\text {com }}$ & $A^{\text {tot }}$ & $B^{\text {fac }}$ & $B^{\text {ca }}$ & \multicolumn{1}{c|}{$B^{\text {tot }}$} & \multicolumn{1}{c}{$\mathscr{B}_{\text {theo }}$} & $\mathscr{B}_{\exp }[4]$ & $\alpha_{\text {theo }}$ & $\alpha_{\text {exp }}$ \\
\hline$\Lambda_{c}^{+} \rightarrow p \bar{K}^{0}$ & 3.45 & 4.48 & 7.93 & -6.98 & -2.06 & -9.04 & $2.11 \times 10^{-2}$ & $(3.18 \pm 0.16) 10^{-2}$ & -0.75 & $0.18 \pm 0.45$ \\
$\Lambda_{c}^{+} \rightarrow \Lambda \pi^{+}$ & 5.34 & 0 & 5.34 & -14.11 & 3.60 & -10.51 & $1.30 \times 10^{-2}$ & $(1.30 \pm 0.07) 10^{-2}$ & -0.93 & $-0.84 \pm 0.09$ \\
$\Lambda_{c}^{+} \rightarrow \Sigma^{0} \pi^{+}$ & 0 & 7.68 & 7.68 & 0 & -11.38 & -11.38 & $2.24 \times 10^{-2}$ & $(1.29 \pm 0.07) 10^{-2}$ & -0.76 & $-0.73 \pm 0.18$ \\
$\Lambda_{c}^{+} \rightarrow \Sigma^{+} \pi^{0}$ & 0 & -7.68 & -7.68 & 0 & 11.34 & 11.34 & $2.24 \times 10^{-2}$ & $(1.25 \pm 0.10) 10^{-2}$ & -0.76 & $-0.55 \pm 0.11$ \\
$\Lambda_{c}^{+} \rightarrow \Xi^{0} K^{+}$ & 0 & -4.48 & -4.48 & 0 & -12.10 & -12.10 & $0.73 \times 10^{-2}$ & $(0.55 \pm 0.07) 10^{-2}$ & 0.90 & $0.77 \pm 0.78$ \\
$\Lambda_{c}^{+} \rightarrow \Sigma^{+} \eta$ & 0 & 3.10 & 3.10 & 0 & -15.54 & -15.54 & $0.74 \times 10^{-2}$ & $(0.53 \pm 0.15) 10^{-2}$ & -0.95 & \\
\hline$\Lambda_{c}^{+} \rightarrow p \pi^{0}$ & 0.41 & -0.81 & -0.40 & -0.87 & 2.07 & 1.21 & $1.26 \times 10^{-4}$ & $<2.7 \times 10^{-4}$ & -0.97 & -0.55 \\
$\Lambda_{c}^{+} \rightarrow p \eta$ & -0.96 & -1.11 & -2.08 & 1.93 & -0.34 & 1.59 & $1.28 \times 10^{-3}$ & $(1.24 \pm 0.29) 10^{-3}$ & -0.75 \\
$\Lambda_{c}^{+} \rightarrow n \pi^{+}$ & 1.64 & -1.15 & 0.50 & -3.45 & 2.93 & -0.52 & $0.91 \times 10^{-4}$ & - & -0.73 \\
$\Lambda_{c}^{+} \rightarrow \Lambda K^{+}$ & 1.66 & -0.08 & 1.58 & -4.43 & 0.55 & -3.70 & $1.07 \times 10^{-3}$ & $(6.1 \pm 1.2) 10^{-4}$ & -0.96 & -0.73 \\
$\Lambda_{c}^{+} \rightarrow \Sigma^{0} K^{+}$ & 0 & 1.49 & 1.49 & 0 & -2.29 & -2.29 & $7.23 \times 10^{-4}$ & $(5.2 \pm 0.8) 10^{-4}$ & -0.73 \\
$\Lambda_{c}^{+} \rightarrow \Sigma^{+} K^{0}$ & 0 & 2.10 & 2.10 & 0 & -3.24 & -3.24 & $1.44 \times 10^{-3}$ & & - & -0.73 \\
\hline \hline
\end{tabular}

for $P$-wave amplitude, two non-perturbative parameters, baryon matrix elements $a_{\mathscr{B} \mathscr{B}}$ and baryonbaryon-meson strong couplings contribute. By using generalized Goldberger-Treiman relation the strong couplings can be reduced to axial-vector form factors $g_{\mathscr{B} \mathscr{B}^{\prime}}^{A}$. Both $a_{\mathscr{B} \mathscr{B}^{\prime}}$ and $g_{\mathscr{B} \mathscr{B}}^{A}$ are calculated in MIT bag model.

\section{Numerical Results}

In this section, we present our numerical results of antitriplet baryon weak decays in following tables. The explicit $S$ - and $P$-wave amplitudes, branching fractions, decay asymmetries as well as comparisons with experiments are summarized in Table 1. The corresponding predictions for $\mathrm{CF}$ and SCS decays of $\Xi_{c}^{+, 0}$ are shown in Table 2 and Table 3, separately.

Table 2: The Cabibbo-favored decays $\Xi_{c} \rightarrow \mathscr{B}_{f} P$ in units of $10^{-2} G_{F} \mathrm{GeV}^{2}$. Branching fractions (in percent) and the up-down decay asymmetry $\alpha$ in theory and experiment are shown in the last four columns.

\begin{tabular}{lcccccc|cccc}
\hline \hline Channel & $A^{\text {fac }}$ & $A^{\text {com }}$ & $A^{\text {tot }}$ & $B^{\text {fac }}$ & $B^{\text {ca }}$ & $B^{\text {tot }}$ & $\mathscr{B}_{\text {theo }}$ & $\mathscr{B}_{\exp }$ & $\alpha_{\text {theo }}$ & $\alpha_{\exp }$ \\
\hline$\Xi_{c}^{+} \rightarrow \Sigma^{+} \bar{K}^{0}$ & 2.98 & -4.48 & -1.50 & -9.95 & 12.28 & 2.32 & 0.20 & - & -0.80 & - \\
$\Xi_{c}^{+} \rightarrow \Xi^{0} \pi^{+}$ & -7.41 & 5.36 & -2.05 & 28.07 & -14.03 & 14.04 & 1.72 & $1.57 \pm 0.83$ & -0.78 & - \\
\hline$\Xi_{c}^{0} \rightarrow \Lambda \bar{K}^{0}$ & -1.11 & -5.41 & -6.52 & 3.66 & 6.87 & 10.52 & 1.33 & - & -0.86 & - \\
$\Xi_{c}^{0} \rightarrow \Sigma^{0} \bar{K}^{0}$ & -2.11 & 3.12 & 1.02 & 7.05 & -9.39 & -2.33 & 0.04 & - & -0.96 & - \\
$\Xi_{c}^{0} \rightarrow \Sigma^{+} K^{-}$ & 0 & -4.42 & -4.42 & 0 & -11.33 & -11.33 & 0.78 & - & 0.98 & - \\
$\Xi_{c}^{0} \rightarrow \Xi^{0} \pi^{0}$ & 0 & -7.58 & -7.58 & 0 & 11.79 & 11.79 & 1.82 & - & -0.77 & - \\
$\Xi_{c}^{0} \rightarrow \Xi^{0} \eta$ & 0 & -10.80 & -10.80 & 0 & -6.17 & -6.17 & 2.67 & - & 0.30 & - \\
$\Xi_{c}^{0} \rightarrow \Xi^{-} \pi^{+}$ & -7.42 & -5.36 & -12.78 & 28.24 & 2.65 & 30.89 & 6.47 & $1.80 \pm 0.52$ & -0.95 & $-0.6 \pm 0.4$ \\
\hline \hline
\end{tabular}

\section{Discussion and Conclusion}

In this work we study Cabibbo-favored (CF) and singly Cabibbo-suppressed (SCS) two-body 
Table 3: The singly Cabibbo-suppressed decays $\Xi_{c} \rightarrow \mathscr{B}_{f} P$ in units of $10^{-2} G_{F} \mathrm{GeV}^{2}$. Branching fractions (in unit of $10^{-3}$ ) and the asymmetry parameter $\alpha$ are shown in the last two columns.

\begin{tabular}{lrrrrrr|rr}
\hline \hline Channel & $A^{\text {fac }}$ & $A^{\text {com }}$ & $A^{\text {tot }}$ & $B^{\text {fac }}$ & $B^{\text {ca }}$ & $B^{\text {tot }}$ & $\mathscr{B}_{\text {theo }}$ & $\alpha_{\text {theo }}$ \\
\hline$\Xi_{c}^{+} \rightarrow \Lambda \pi^{+}$ & 0.46 & -1.50 & -1.04 & -1.69 & 2.16 & 0.47 & 0.85 & -0.33 \\
$\Xi_{c}^{+} \rightarrow \Sigma^{0} \pi^{+}$ & -0.90 & -1.00 & -1.90 & 3.29 & 0.74 & 4.03 & 4.30 & -0.95 \\
$\Xi_{c}^{+} \rightarrow \Sigma^{+} \pi^{0}$ & 0.32 & 0.99 & 1.32 & -1.16 & 1.61 & 0.44 & 1.36 & 0.23 \\
$\Xi_{c}^{+} \rightarrow \Sigma^{+} \eta$ & -0.74 & 1.42 & 0.68 & 2.58 & -2.19 & 0.39 & 0.32 & 0.36 \\
$\Xi_{c}^{+} \rightarrow p \bar{K}^{0}$ & 0 & -2.10 & -2.10 & 0 & 2.64 & 2.64 & 3.96 & -0.83 \\
$\Xi_{c}^{+} \rightarrow \Xi^{0} K^{+}$ & -2.30 & 1.16 & -1.14 & 8.43 & -3.46 & 4.97 & 2.20 & -0.98 \\
\hline$\Xi_{c}^{0} \rightarrow \Lambda \pi^{0}$ & -0.12 & 1.06 & 0.95 & 0.42 & -0.96 & -0.53 & 0.24 & -0.41 \\
$\Xi_{c}^{0} \rightarrow \Lambda \eta$ & 0.27 & 1.51 & 1.78 & -0.94 & -0.27 & -1.20 & 0.77 & -0.45 \\
$\Xi_{c}^{0} \rightarrow \Sigma^{0} \pi^{0}$ & -0.23 & -0.70 & -0.93 & 0.82 & 1.36 & 2.18 & 0.38 & -0.98 \\
$\Xi_{c}^{0} \rightarrow \Sigma^{0} \eta$ & 0.53 & -1.00 & -0.48 & -1.83 & 1.55 & -0.28 & 0.05 & 0.36 \\
$\Xi_{c}^{0} \rightarrow \Sigma^{-} \pi^{+}$ & -1.28 & -1.41 & -2.69 & 4.67 & 0.22 & 4.89 & 2.62 & -0.90 \\
$\Xi_{c}^{0} \rightarrow \Sigma^{+} \pi^{-}$ & 0 & 1.41 & 1.41 & 0 & 2.49 & 2.49 & 0.71 & 0.89 \\
$\Xi_{c}^{0} \rightarrow p K^{-}$ & 0 & -0.94 & -0.94 & 0 & -1.86 & -1.86 & 0.35 & 0.99 \\
$\Xi_{c}^{0} \rightarrow n \bar{K}^{0}$ & 0 & -2.10 & -2.10 & 0 & 2.96 & 2.96 & 1.40 & -0.89 \\
$\Xi_{c}^{0} \rightarrow \Xi^{0} K^{0}$ & 0 & 2.10 & 2.10 & 0 & -4.17 & -4.17 & 1.32 & -0.85 \\
$\Xi_{c}^{0} \rightarrow \Xi^{-} K^{+}$ & -2.31 & -0.94 & -3.24 & 8.49 & 0.71 & 9.20 & 3.90 & -0.97 \\
\hline \hline
\end{tabular}

hadronic weak decays of the antitriplet charmed baryons $\Lambda_{c}^{+}, \Xi_{c}^{0}$ and $\Xi_{c}^{+}$with more focus on the last two. To estimate the nonfactorizable contribution, we work in the pole model with the currentalgebra technique. Throughout the whole calculations, all the non-perturbative parameters, including form factors, baryon matrix elements and axial-vector form factors are evaluated using the MIT bag model. We give predictions of branching fractions and decay asymmetries in the end.

The long-standing puzzle with the branching fraction and decay asymmetry of $\Lambda_{c}^{+} \rightarrow \Xi^{0} K^{+}$ is resolved. The predicted $\mathscr{B}\left(\Xi_{c}^{+} \rightarrow \Xi^{0} \pi^{+}\right)$agrees well with the measurement inferred from Belle and CLEO, while the calculated $\mathscr{B}\left(\Xi_{c}^{0} \rightarrow \Xi^{-} \pi^{+}\right)$is too large compared to the recent Belle measurement. Owing to large cancellation between factorizable and nonfactorizable contributions, the rate of $\Lambda_{c}^{+} \rightarrow n \pi^{+}$is found to be of the same order as that of $\Lambda_{c}^{+} \rightarrow p \pi^{0}$. It is important to measure both SCS modes to understand their underlying mechanism.

\section{References}

[1] H. Y. Cheng and B. Tseng, Phys. Rev. D 48, 4188 (1993) [hep-ph/9304286].

[2] H. Y. Cheng, X. W. Kang and F. Xu, Phys. Rev. D 97, no. 7, 074028 (2018) [arXiv:1801.08625 [hep-ph]].

[3] J. Zou, F. Xu, G. Meng, and H. .Y. Cheng, [arXiv:1910.13626[hep-ph]].

[4] M. Tanabashi et al. [Particle Data Group], Phys. Rev. D 98, no. 3, 030001 (2018). doi:10.1103/PhysRevD.98.030001 